\title{
Optics for the Imaging X-ray Polarimetry
}

\section{Explorer}

\section{Brian Ramsey NASA/MSFC}

\author{
For the IXPE Team
}




\section{The IMAging X-RAY Polarimetry EXPLORER IXPE}

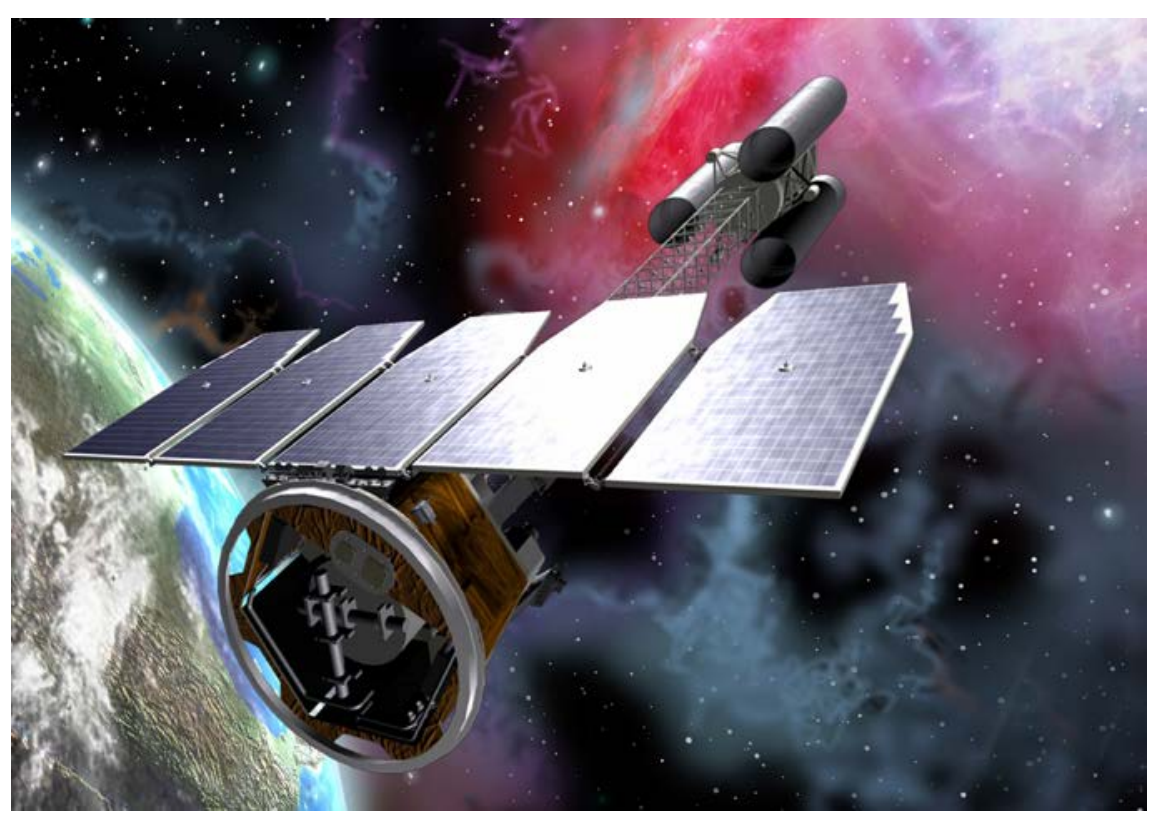

IXPE is a NASA Small Explorer Mission dedicated to X-ray polarimetry the first of its kind - opening up the field of imaging polarimetry

It was selected in January 2017 for a flight in 2020/2021 


\section{IXPE ADDRESSES KEY SCIENTIFIC OBJECTIVES}

- Addresses key questions, providing new scientific results and constraints that trace back to the Astrophysics Roadmap and the Decadal Survey

- What is the spin of a black hole?

- What are the geometry and magnetic-field strength in magnetars?

- Was our Galactic Center an Active Galactic Nucleus in the recent past?

- What is the magnetic field structure in synchrotron X-ray sources?

- What are the geometries and origins of $X$-rays from pulsars (isolated and accreting)?

- Provides powerful and unique capabilities

- Reduces integration time by a factor of 100 over our OSO-8 experiment

- Simultaneously provides imaging, energy, timing, and polarization data

- Devoid of instrument systematic effects at less than a fraction of a percent

- Meaningful polarization measurements for a large number of sources of different classes, as evidenced by our Design Reference Mission 


\section{IXPE TEAM AND Mission}

Institutional Roles and Responsibilities

\begin{tabular}{|c|c|c|}
\hline \multirow{2}{*}{$\begin{array}{l}\text { PI team, project management, } \\
\text { SE and S\&MA oversight, mirror } \\
\text { module fabrication, X-ray } \\
\text { calibration, science operations, } \\
\text { and data analysis and archiving }\end{array}$} & \multicolumn{2}{|c|}{ 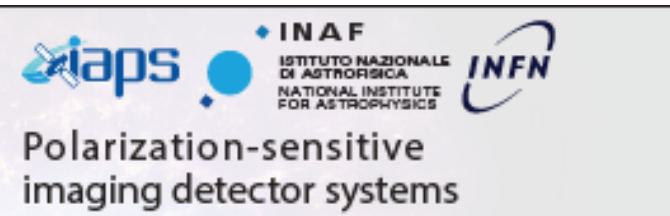 } \\
\hline & \multicolumn{2}{|c|}{ (2) LAP Mission operations } \\
\hline \multirow{2}{*}{$\begin{array}{l}\text { Detector system } \\
\text { funding, ground station }\end{array}$} & \multicolumn{2}{|c|}{ Stanford Scientific theory } \\
\hline & McGill & $\begin{array}{l}\text { Science Working } \\
\text { Group Co-Chair }\end{array}$ \\
\hline Buele $\begin{array}{l}\text { Spacecraft, payload } \\
\text { structure, payload, } \\
\text { observatory I \&T }\end{array}$ & 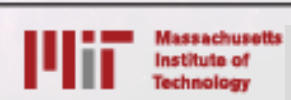 & Col \\
\hline
\end{tabular}

\section{Mission Design and Operations}

- Pegasus XL launch from Kwajalein

- 540-km circular orbit at $0^{\circ}$ inclination

- 2 year baseline mission, 1 year SEO

- Point-and-stare at known targets

- Science Operations Center at MSFC

- Mission Operations Center at CU/LASP

- Malindi ground station (Singapore Backup)

- Launch ready by end of 2020 


\section{Payload Overview}

Set of three mirror module assemblies (MMA) focus $x$ rays onto three corresponding focal plane detector units

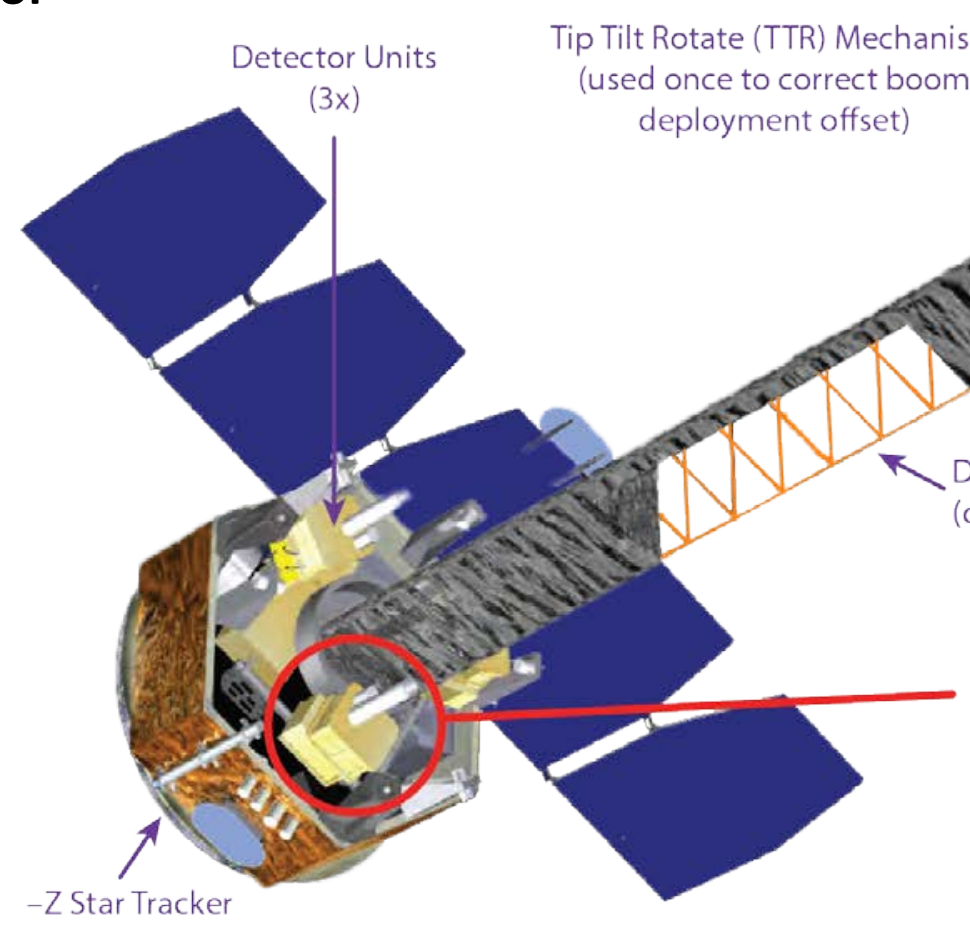

Mirror modules provide imaging and background reduction

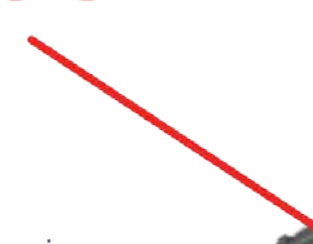




\section{MiRROR MOdULE ASSEMBLY}
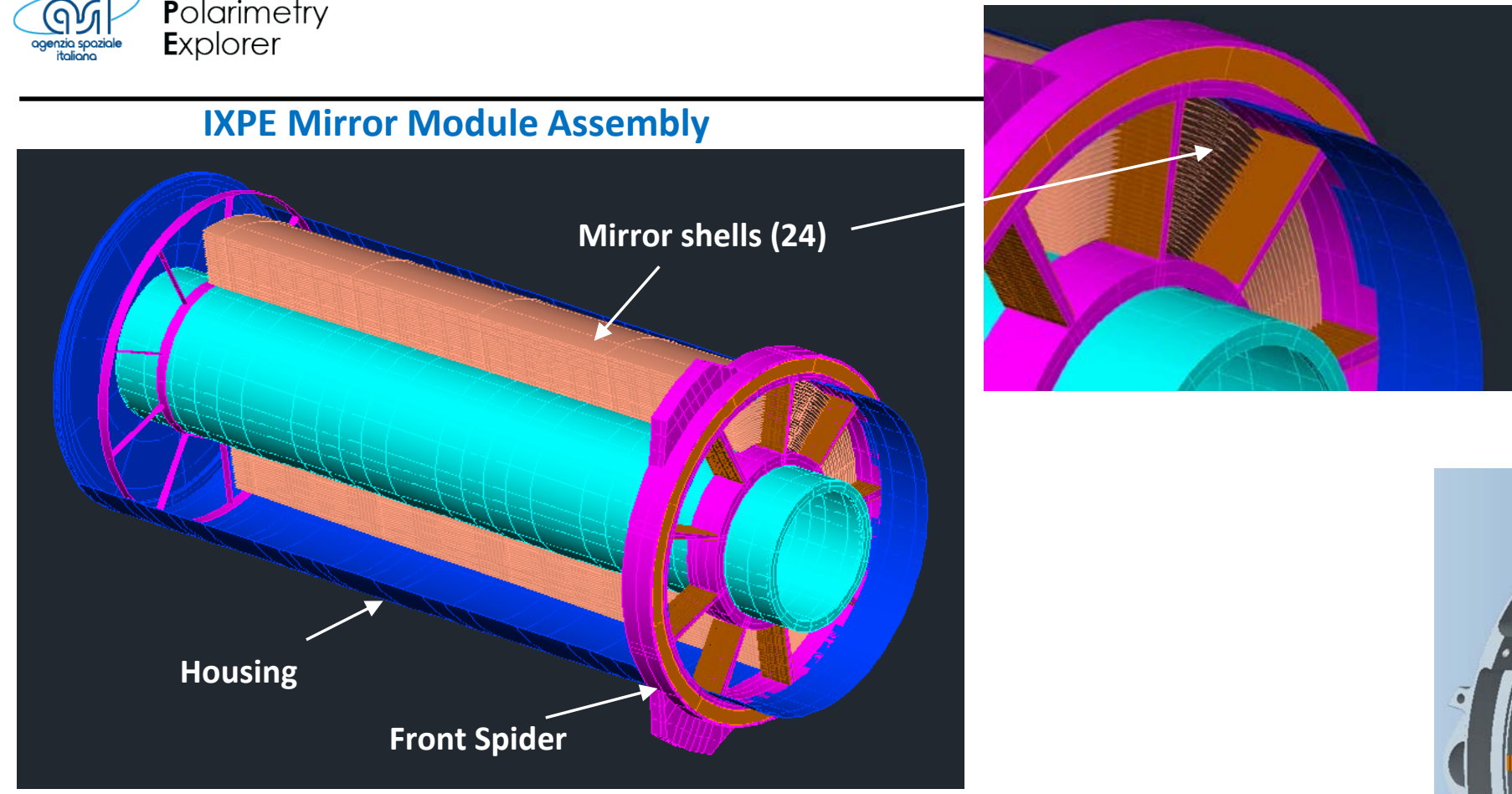

\section{Design approach}

- Uses a single rigid spider to support the 24 nested shells and attach module to structure.

- Light weight housing mainly for thermal control

- Limit (rear) spider does not support mirror shells but limits their vibrations during launch.

- Mounting combs provide shell attachment points

Shell Mounting Comb

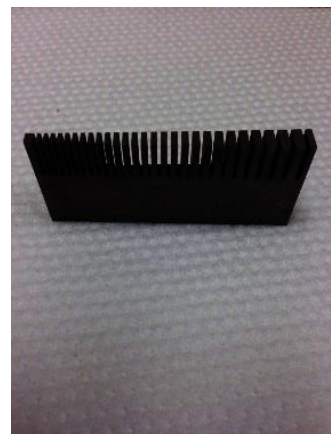

Front Spider

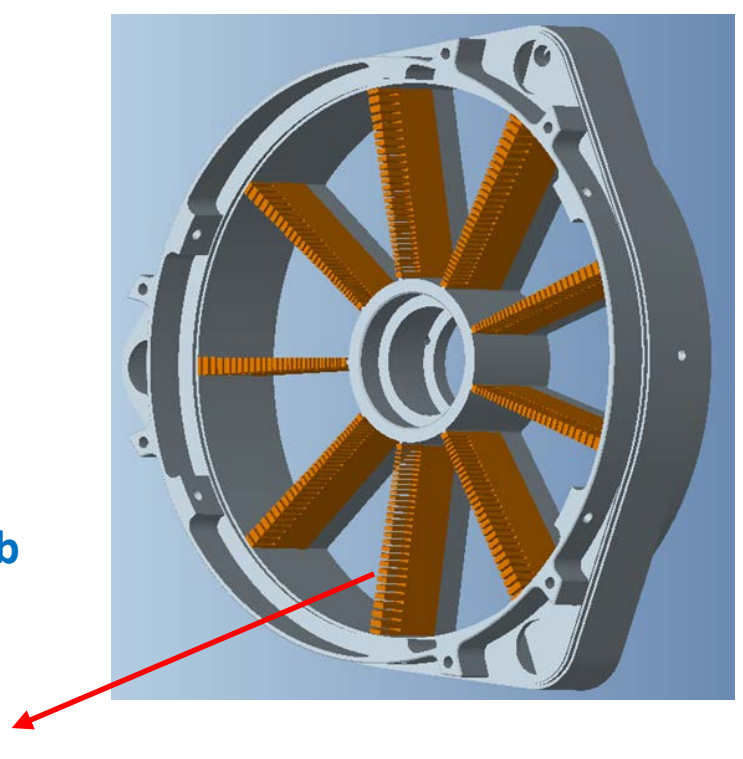




\section{MirRor Shell Fabrication - Electroformed Replication}

\section{Mandrel Fabrication}

1. Machine mandrel from aluminum bar

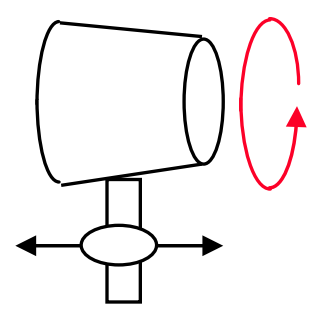

2. Coat mandrel with electroless nickel (NiP)

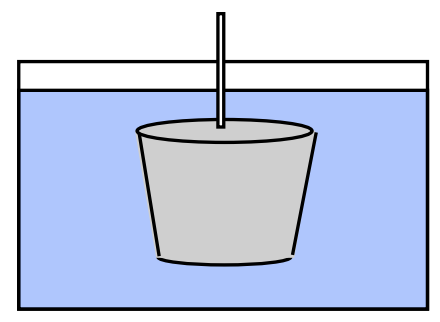

3. Diamond turn mandrel for submicron figure

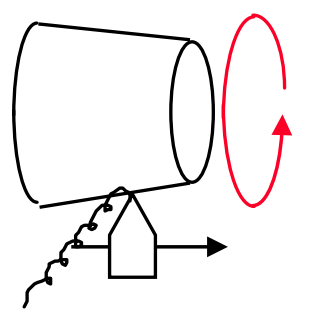

4. Polish mandrel to $0.3-0.4 \mathrm{~nm}$ rms

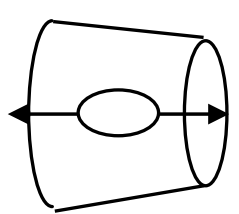

5. Metrology on mandrel

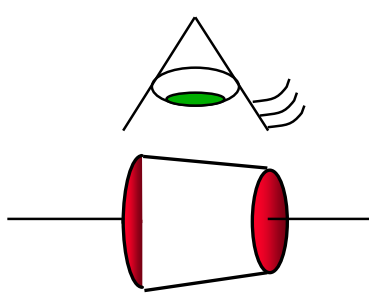

\section{Mirror Shell Fabrication}

6. Passivate mandrel surface to reduce shell adhesion

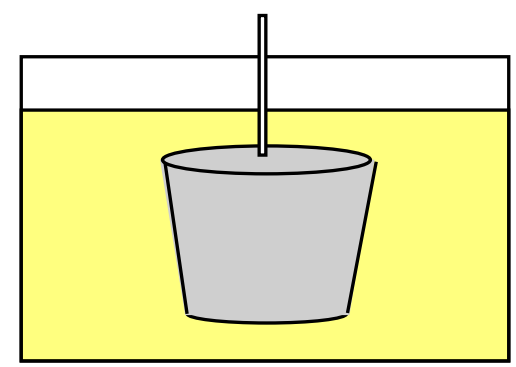

7. Electroform Nickel/Cobalt shell on to mandrel

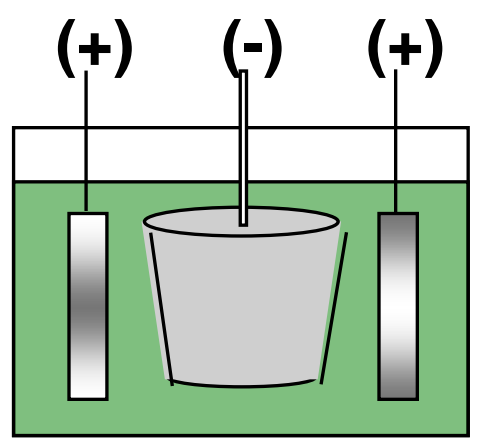

8. Separate shell from mandrel in cold water bath

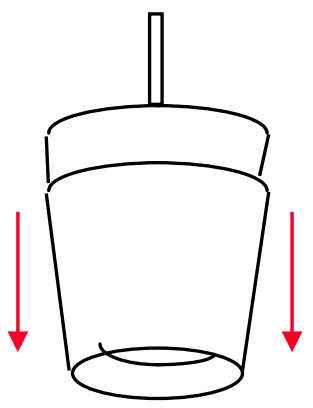

NiCo electroformed mirror shells

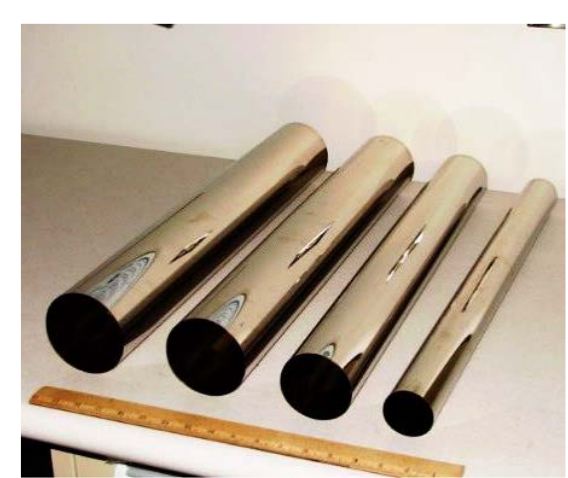




\section{MSFC INFRASTRUCTURE FOR X-RAY OPTICS FABRICATION}

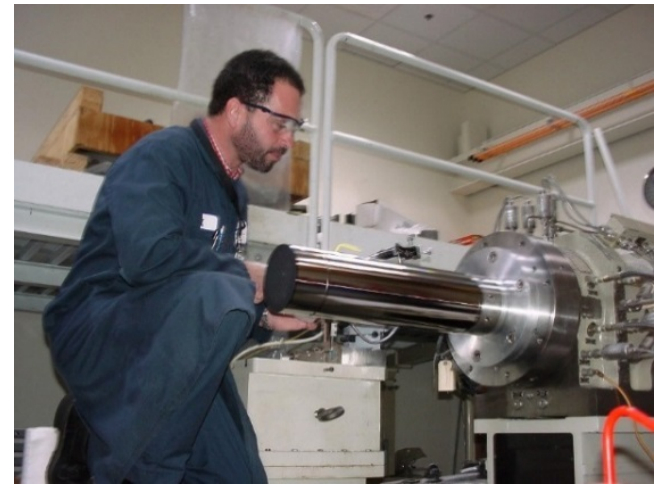

Mandrel diamond turning

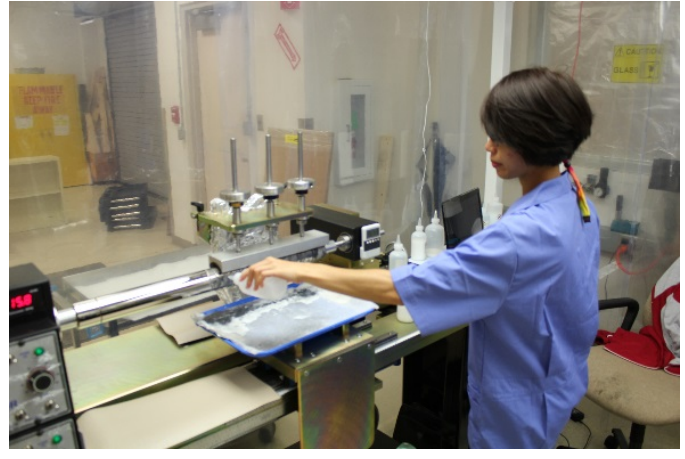

Mandrel polishing

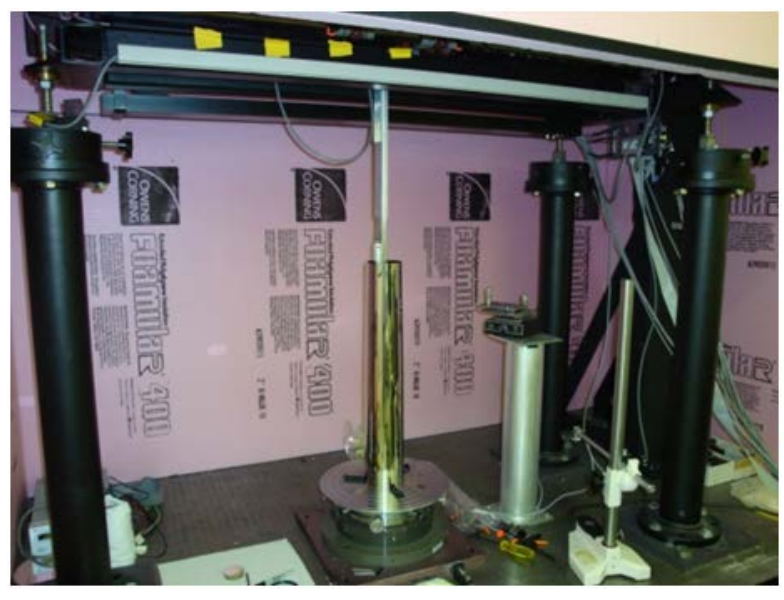

Mandrel and shell metrology

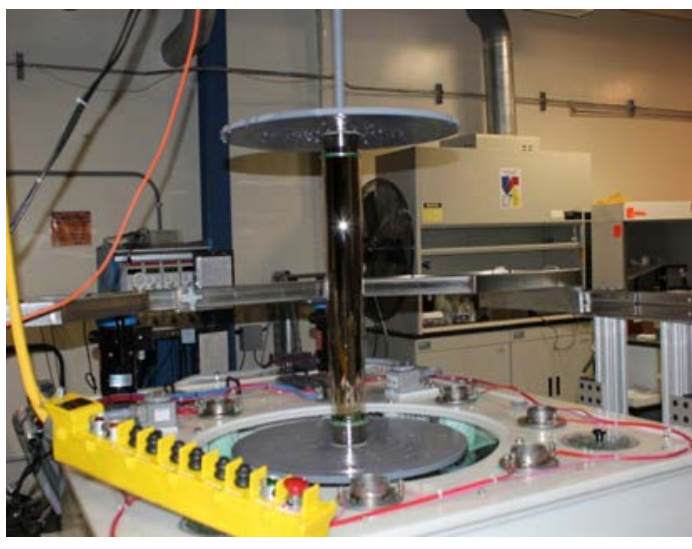

Nickel/Cobalt shell electroforming

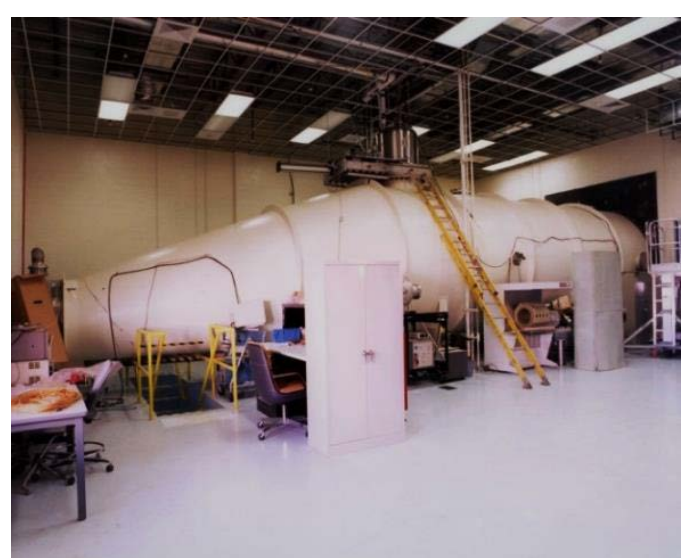

X-ray testing and calibration 


\section{MirRor SheLl INTEGRATION AND AlignMENT}

- Shell assembly proceeds from the inner shell outwards

- The assembly system holds each successive shell on a system of wires that can be moved radially and adjusted in tension.

- Keyence proximity sensors rotate around the hanging shell and measure radial displacements of the mirror external surface

- Software takes the displacement data as a function of rotation angle and fits various curves (and calculate various performance parameters) to aid in the alignment process.

- When shell performance is satisfactory, it is glued into the spider comb and the next shell is mounted

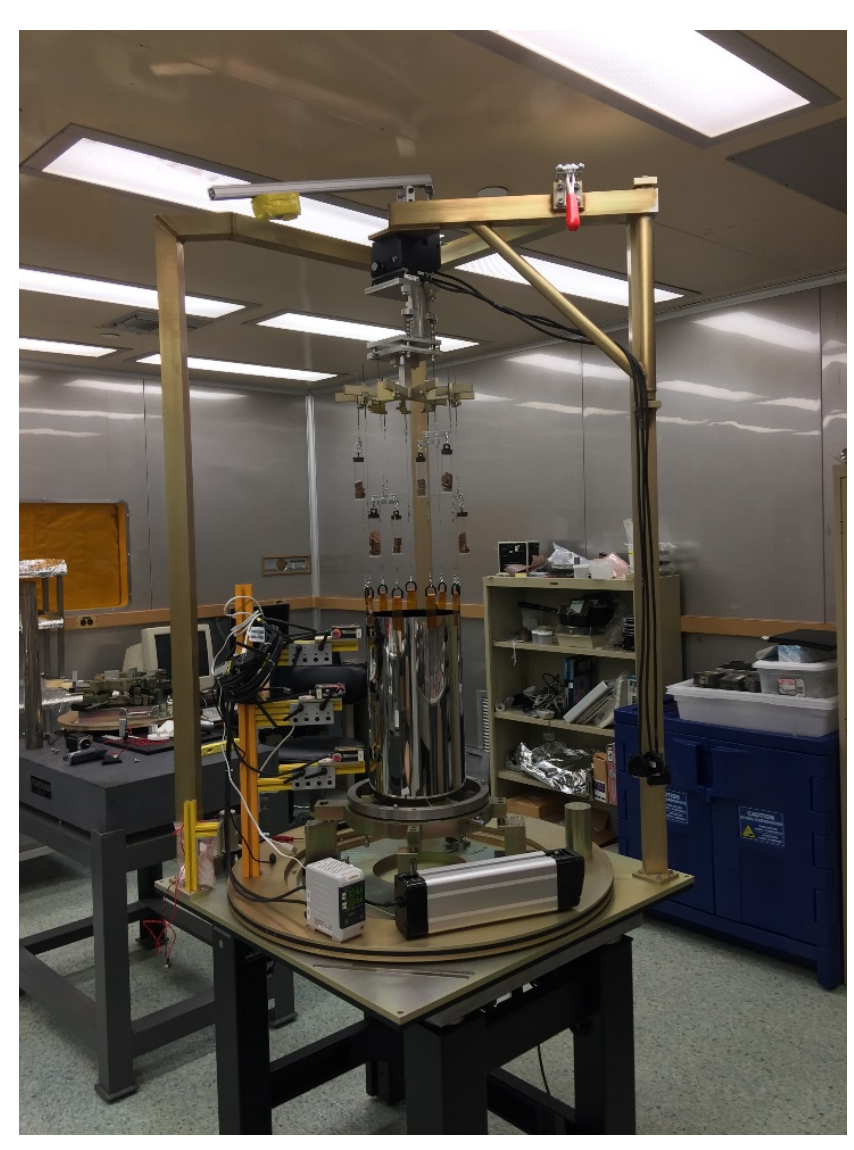

Integration System 


\section{Mirror Module Assembly Properties}

\begin{tabular}{|l|l|}
\hline Property & Value \\
\hline Number of modules & 3 \\
\hline Mirror shells per module & 24 \\
\hline Inner, outer shell diameter & $162,272 \mathrm{~mm}$ \\
\hline Total shell length & $600 \mathrm{~mm}$ \\
\hline Inner, outer shell thickness & $180,260 \mu \mathrm{m}$ \\
\hline Shell material & Nickel cobalt alloy \\
\hline Effective area per module & $210 \mathrm{~cm}$ (2.3 keV) \\
\hline Angular resolution & $>230 \mathrm{~cm} 2$ (3-6 keV) \\
\hline Detector limited FOV & $\leq 25$ arcsec HPD \\
\hline Focal length & 12.9 arcmin \\
\hline Mass (3 assemblies) & $4 \mathrm{~m}$ \\
\hline contingency
\end{tabular}

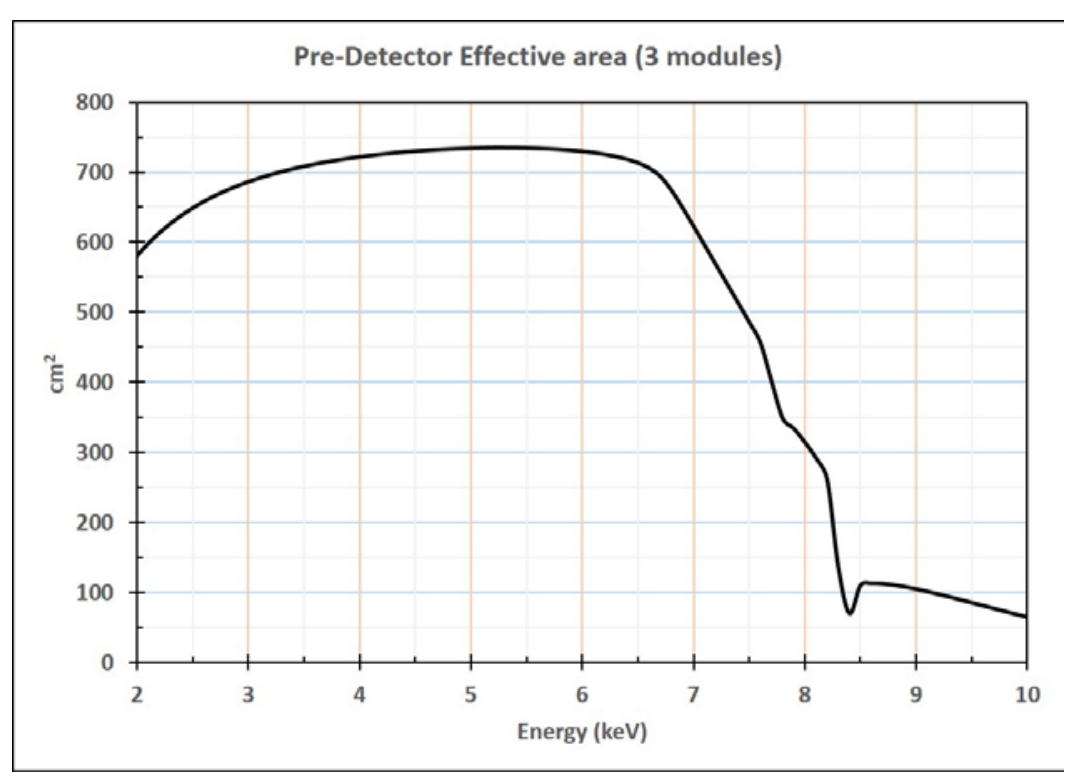




\section{GHOST RAY ANALYSIS}

- Radiation from outside the instrument field of view can reflect off the mirror shells, end up in the detector, and constitute a background

- This typically arises from single reflections from either the hyperbolic or parabolic segment of the mirror (depends on exact module geometry)

- For IXPE, only single reflections from the hyperbola (H singles) contribute

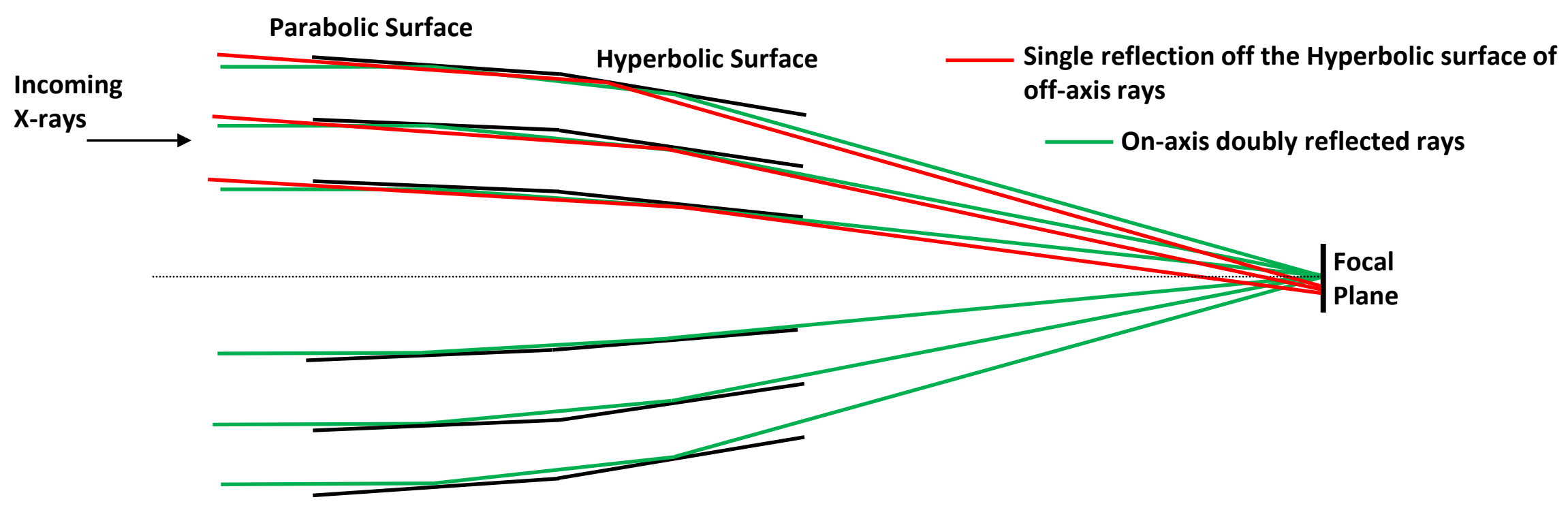




\section{GHOSt RAY ANALYSIS}

- Ray trace analysis was performed

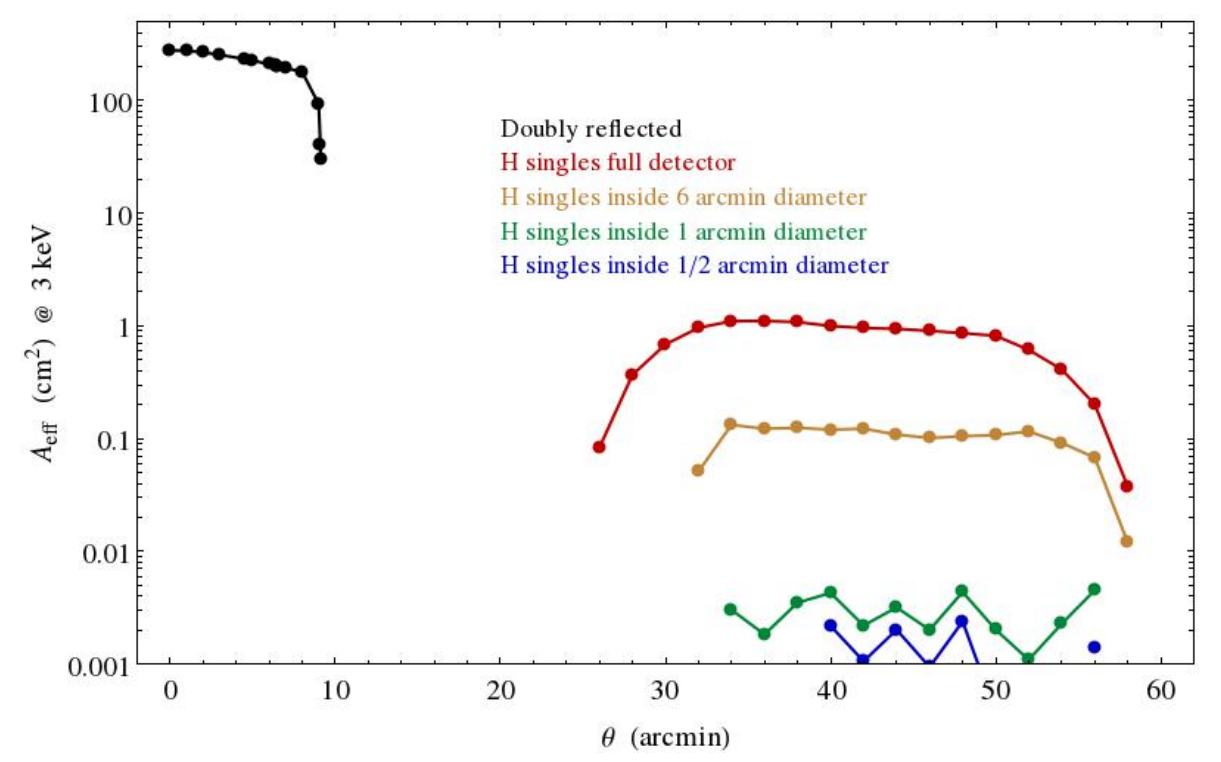

- Results of IXPE MMA stray radiation analysis:

- Stray radiation reaching detector is from a small annulus of the sky from 25 arcminutes off axis to just under 60 arcmin.

- Peak magnitude (effective area) is $~ 300 \times$ lower than the on-axis signal, integrated over the whole detector.

- Imaging further reduces this by a large amount (see above figure) 


\section{GHOSt RAY ANALYSIS}

- Are there any very bright sources within 25 to 60 arcminute of an intended target that could increase the background for that observation?

- Not a problem for point sources as stray radiation is reduced by more than factor of $10^{5}$

- What about extended sources ?

- Use the MAXI (Monitor of All-sky X-ray Image) catalog of sources, appropriate for the IXPE energy range, to search around IXPE design reference mission targets

- The only source affected is SGR B2, due to the bright source SAXJ1747-285 nearby. However, the imaging properties of IXPE will isolate this stray radiation at the edge of the detector, away from the target. 


\section{THERMAL REQUIREMENTS}

- Thermal requirements derived from FEA analysis and subsequent ray tracing.

- Looked at temp variations across mirror diameter and along axis.

- Most sensitive to temp gradients from one side of mirror to other

- Set requirements of max $2{ }^{\circ} \mathrm{C}$ variation across mirror assembly

- Achieved with $\sim 10 \mathrm{~W}$ orbit average power per module

Example of mirror shell distortions for $2^{\circ} \mathrm{C}$ change across mirror diameter

Affect of this on mirror shell performance

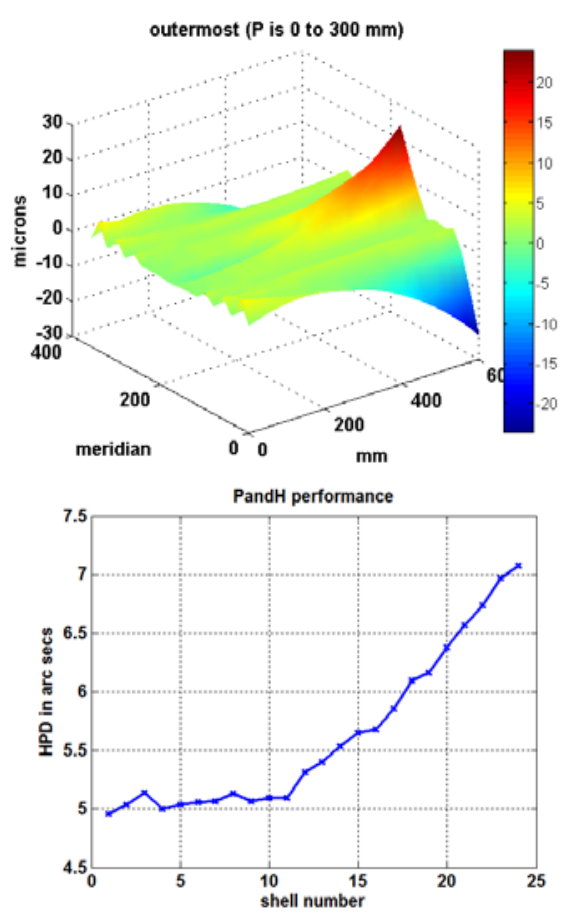

Thermal model of mirror module assembly.

Entrance and exit aperture thermal shields: 1.4 micron polyimide coated with 300 $A$ aluminum 


\section{X-RAY SHIELDS}

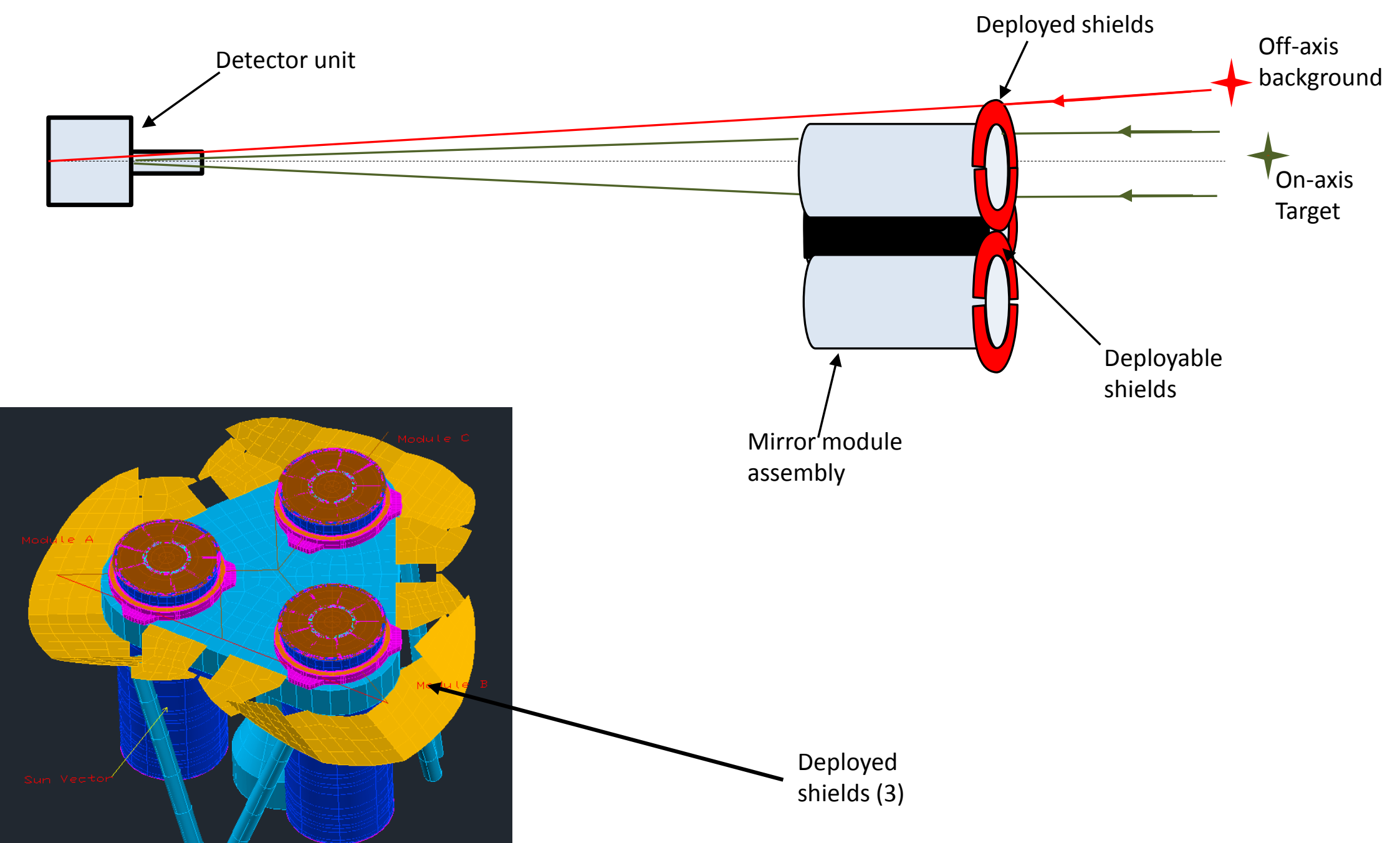




\section{ON-ORBIT ALIGNMENT}

- If after deployment the Mirror Module Assemblies (MMAs) are out in translation, we would use a lookup table to tell us which tip/tilts to actuate and the amount of adjustment to apply

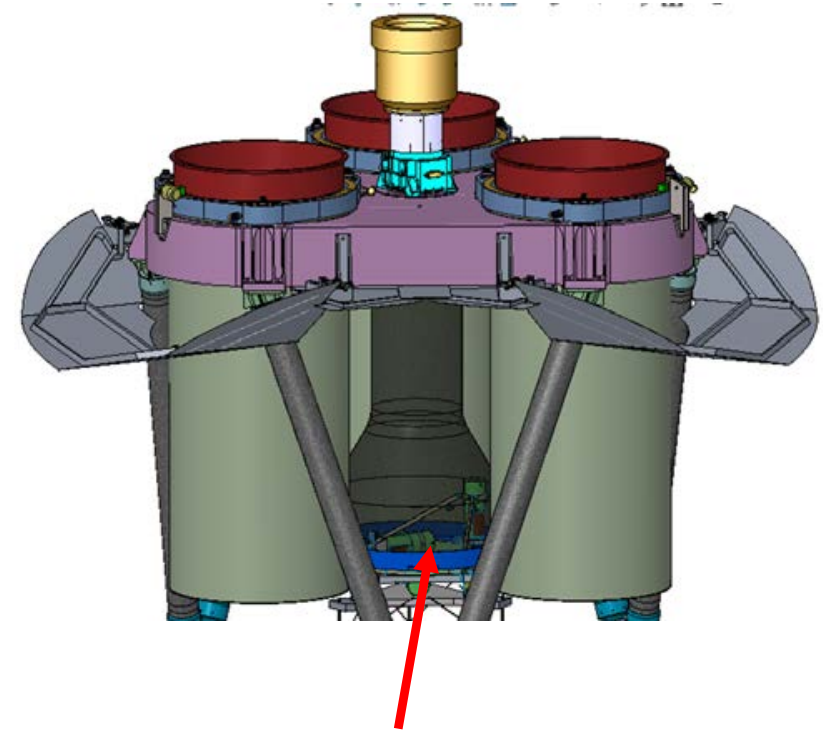

Tip/tilt/rotate adjustment (on top of extending boom)

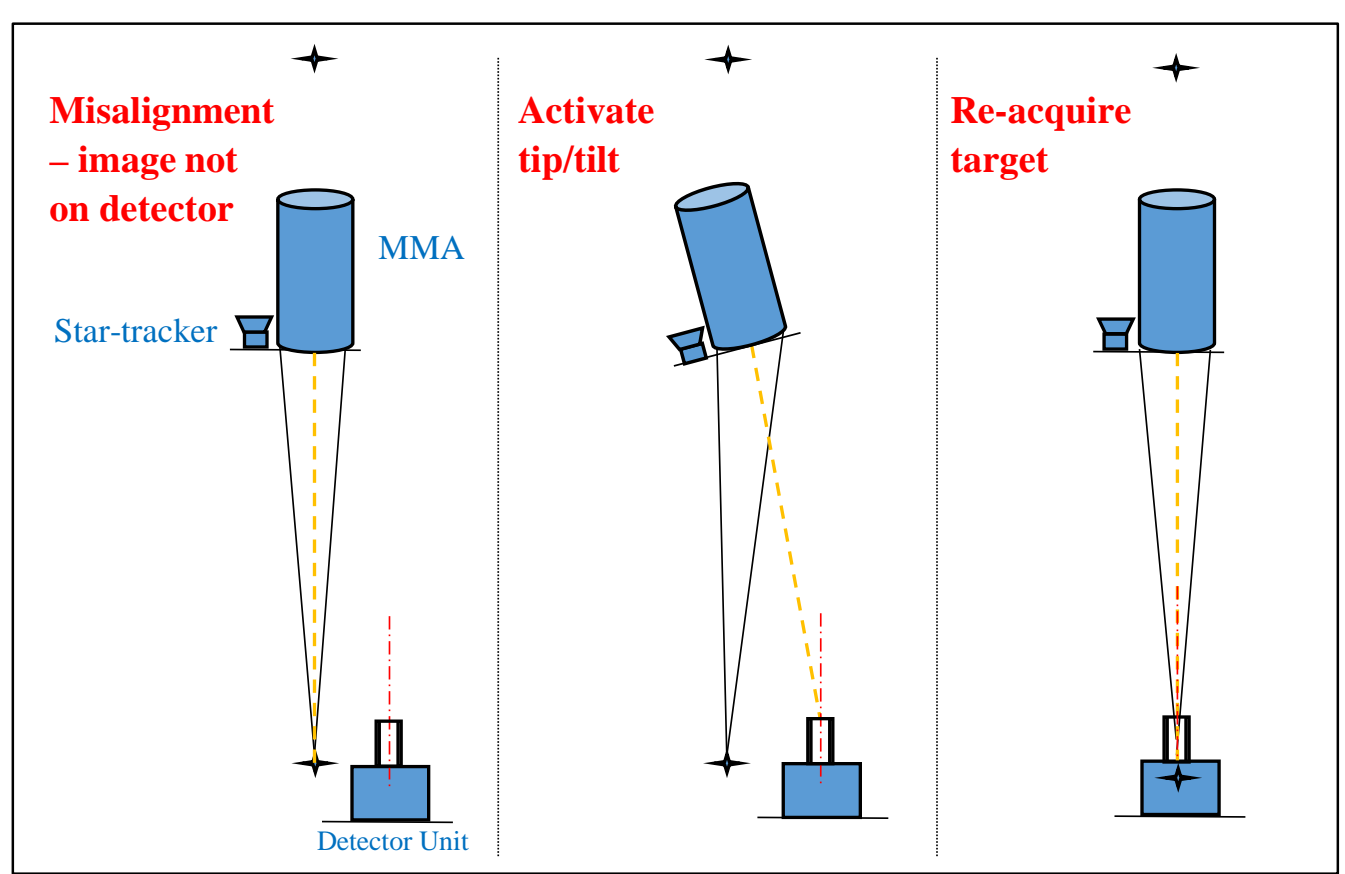




\section{MMA ACTIVITIES}

- Build and test an engineering unit (in progress, completion in March 2018)

- Will have a subset of flight mirror shells

- Will be used to:

- Exercise the whole fabrication and assembly process

- Test all handling fixtures

- Confirm the mechanical design through environmental testing

- Provide a test system to verify procedures and hardware for the MMA and end-to-end ground calibration (along with a detector engineering unit).

- Build and test 4 flight units

- 3 Flight and 1 spare unit

- Will all go through acceptance-level vibration tests

- Will all be fully calibrated

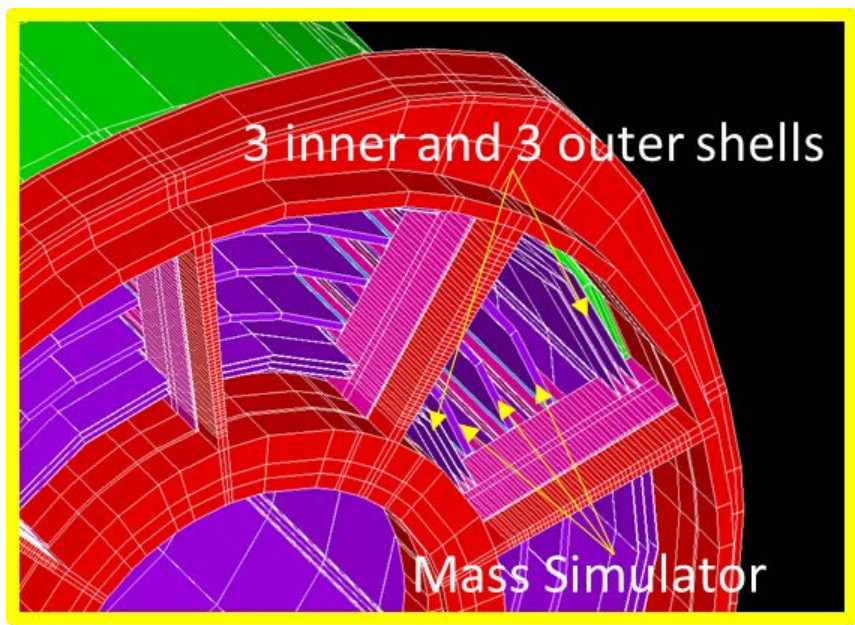




\section{X-ray Calibration}

\section{- Plan}

- Calibrate Detector Units (DU) in Italy at INAF/IAPS

- Calibrate Mirror Module Assemblies (MMA) at MSFC

- Perform end to end calibration at MSFC

- Preparations with engineering units of MMA and DU

- Allows test of SOC systems

\section{- Calibration Heritage}

- MSFC: Chandra, ART-XC, FOXSI and HERO calibration

- Italy: SXRP, BeppoSAX, Super AGILE, Fermi/LAT

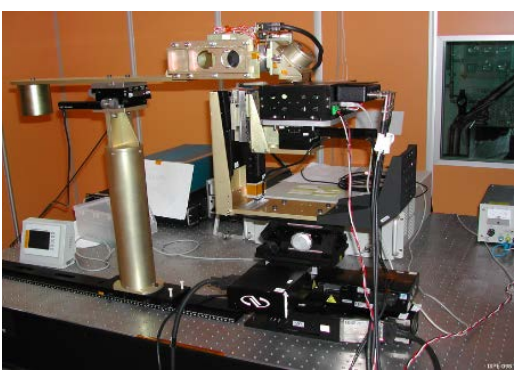

Custom polarized sources for DU calibrations at IAPS
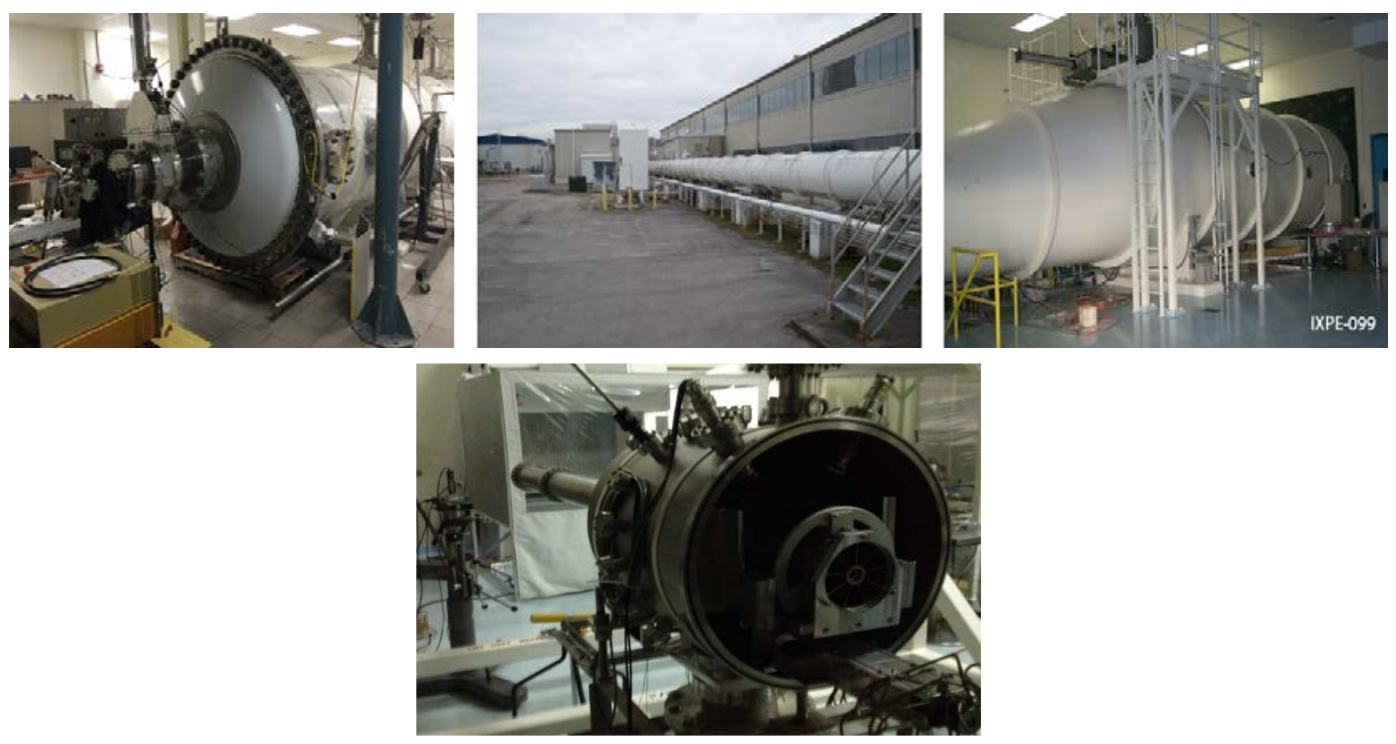

Stray-light test facility at MSFC 


\section{IXPE SCHEDULE (UNDER REVIEW)}

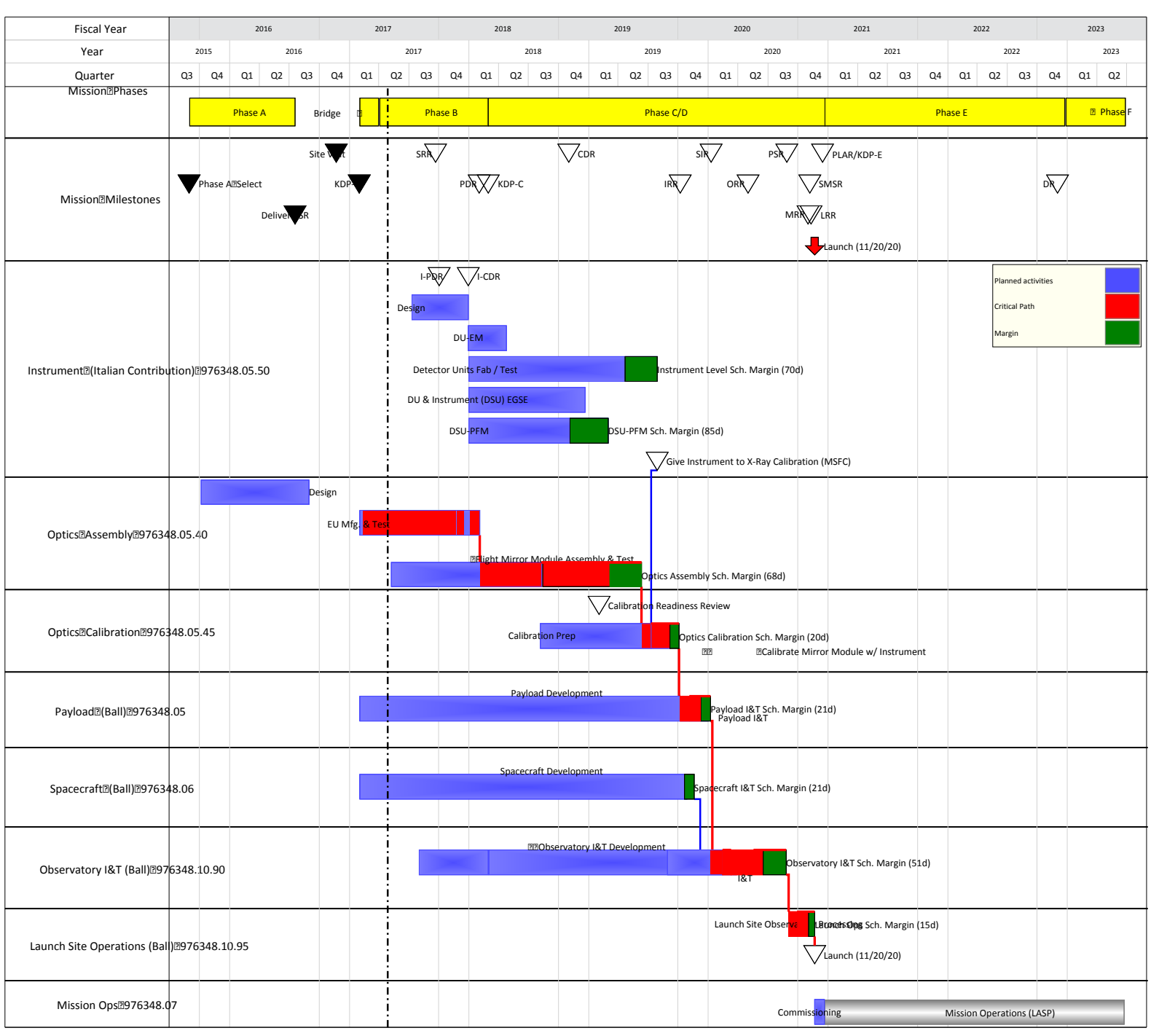

